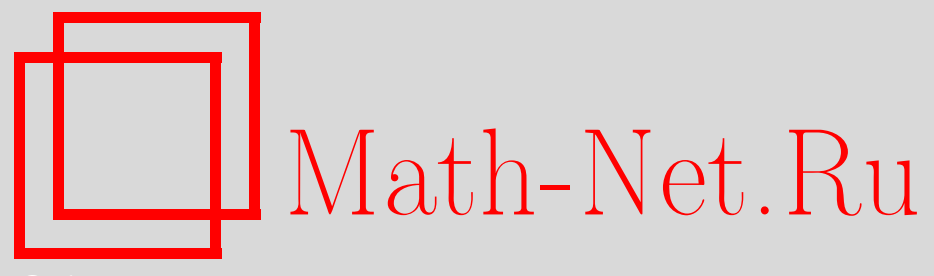

И. А. Икромов, Суммируемость осцилляторных интегралов по параметрам и проблема об ограничении преобразования Фурье на кривых, Матем. заметки, 2010, том 87, выпуск 5, 734-755

DOI: https://doi.org/10.4213/mzm4619

Использование Общероссийского математического портала Math-Net.Ru подразумевает, что вы прочитали и согласны с пользовательским соглашением http://www . mathnet.ru/rus/agreement

Параметры загрузки:

IP: 54.164 .48 .24

26 апреля 2023 г., 16:49:23

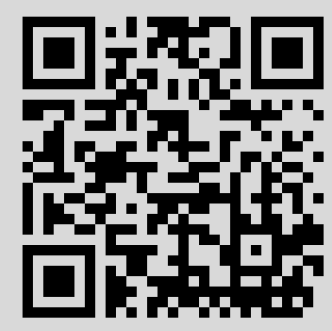


Том 87 выпуск 5 май 2010

УДК 517.518 .5

\section{Суммируемость осцилляторных интегралов по параметрам и проблема об ограничении преобразования Фурье на кривых}

\section{И. А. Икромов}

В работе получено решение задачи о точном показателе суммируемости преобразования Фурье зарядов, сосредоточенных на дифференцируемых кривых конечного типа. Исследовано поведение осцилляторных интегральных операторов, связанных с преобразованием Фурье зарядов, сосредоточенных на кривых. Приведены необходимые и достаточные условия ограниченности преобразования Фурье на гладких кривых конечного типа.

Библиография: 23 названия.

1. Постановка задачи. Пусть $\gamma \subset \mathbb{R}^{n}$ - дифференцируемая кривая в $\mathbb{R}^{n}$ с параметризацией $\varphi:[-1,1] \mapsto \mathbb{R}^{n}$ и $\psi \in C_{0}^{\infty}\left(\mathbb{R}^{n}\right)$ - фиксированная гладкая функция. Рассмотрим заряд, заданный в виде $d \mu_{\alpha}:=\left(\varphi^{\prime}, \varphi^{\prime}\right)^{\alpha} \psi(\varphi(t)) d t$, где $\alpha$ - вещественное число, удовлетворяющее условию

$$
\int_{\mathbb{R}}\left(\varphi^{\prime}(t), \varphi^{\prime}(t)\right)^{\alpha}|\psi(\varphi(t))| d t<\infty .
$$

Отметим, что при $\alpha \neq 1 / 2$ заряд $d \mu_{\alpha}$ зависит от выбора параметризации и является относительно-инвариантным зарядом группы диффеоморфизмов отрезка $[-1,1]$.

Преобразование Фурье этого заряда определяется по формуле

$$
\widehat{d \mu_{\alpha}}(\xi)=\int_{\mathbb{R}} e^{2 \pi i(\xi, \varphi(t))}\left(\varphi^{\prime}(t), \varphi^{\prime}(t)\right)^{\alpha} \psi(\varphi(t)) d t .
$$

Рассматривается задача: найти

$$
q(\gamma, \alpha):=\inf \left\{q \in[1,+\infty]: \widehat{d \mu_{\alpha}} \in L^{q}\left(\mathbb{R}^{n}\right)\right\}
$$

где $L^{q}\left(\mathbb{R}^{n}\right)$ - пространство интегрируемых функций со степенью $q, 1 \leqslant q<\infty$; в случае $q=\infty$ мы имеем дело с пространством существенно ограниченных функций.

ЗАмечАниЕ 1.1. Вообще говоря, возможен случай $q(\gamma, \alpha)=\infty$. Например, если $\gamma \subset \mathbb{R}^{3}$ - любая плоская кривая и $d \mu_{\alpha}$ - любая ненулевая мера на этой кривой, то $\widehat{d \mu_{\alpha}}(\xi)$ не принадлежит $L^{q}\left(\mathbb{R}^{3}\right)$ при любом $q<\infty$.

Работа выполнена при подержке Госкомитета науки и техники Республики Узбекистан (грант OT-Ф1-006).

(С) И. А. Икромов, 2010 
2. Краткая история проблемы. Аналогичная проблема суммируемости для тригонометрического интеграла

$$
J(\xi):=\int_{0}^{1} e^{2 \pi i P(t, \xi)} d t, \quad \text { где } \quad P(t, \xi):=\xi_{1} t+\xi_{2} t^{2}+\cdots+\xi_{n} t^{n},
$$

была рассмотрена Виноградовым [1]. Он получил следующую оценку для интеграла $J(\xi)$ :

$$
|J(\xi)| \leqslant \min \left\{1,32|\xi|_{\infty}^{-1 / n}\right\}, \quad \text { где } \quad|\xi|_{\infty}:=\max _{1 \leqslant j \leqslant n}\left|\xi_{j}\right| .
$$

Из оценки Виноградова следует суммируемость интеграла $J(\xi)$ со степенью $q>n^{2}$.

В 1952 г. Хуа Ло-Ген [2] доказал принадлежность тригонометрического интеграла $J(\xi)$ к $L^{q}\left(\mathbb{R}^{n}\right)$ при $q>n^{2} / 2+n$.

Далее, в работе [3] Архипова, Карацубы и Чубарикова доказано, что при любом $q>n(n+1) / 2+1$ тригонометрический интеграл $J(\xi)$ принадлежит $L^{q}\left(\mathbb{R}^{n}\right)$ и при $q \leqslant$ $n(n+1) / 2+1$ он не принадлежит $L^{q}\left(\mathbb{R}^{n}\right)$. Более того, если $J(\xi)$ - тригонометрический интеграл с фазовой функцией

$$
P(t, \xi):=\xi_{1} t^{k_{1}}+\xi_{2} t^{k_{2}}+\cdots+\xi_{n} t^{k_{n}},
$$

где $k_{1}<k_{2}<\cdots<k_{n}$ - натуральные числа такие, что $q_{0}:=k_{1}+k_{2}+\cdots+k_{n}>$ $n(n+1) / 2$, то при любом $q>q_{0}$ тригонометрический интеграл $J(\xi)$ принадлежит $L^{q}\left(\mathbb{R}^{n}\right)$ и при $q \leqslant q_{0}$ он не принадлежит $L^{q}\left(\mathbb{R}^{n}\right)$.

Заметим, что в случае $q_{0}=n(n+1) / 2$ точный показатель суммируемости тригонометрического интеграла равняется $q_{0}+1$, а в случае $q_{0}>n(n+1) / 2$ он совпадает с $q_{0}$. Такое явление естественно наблюдается и в общем случае.

Задача о точном показателе суммируемости кратных тригонометрических интегралов с полиномиальной фазой до сих пор остается открытой. Известны некоторые результаты о конечности показателя суммируемости, полученные в работе [3] (также см. [4]).

3. Мотивы проблемы. Одной из нерешенных проблем гармонического анализа является задача об ограничении преобразования Фурье на множествах меры нуль. Известны некоторые результаты об ограничении преобразования Фурье на гладких поверхностях (более подробное обсуждение известных результатов содержится в [5], [6]).

В 1996 г. Мокенхаупт [7] показал связь между задачей о точном показателе суммируемости тригонометрических интегралов и проблемой об ограничении преобразования Фурье на гладких поверхностях. В работах Крист [8], Друри [9] рассмотрена задача об ограниченности следующего оператора сужения: $R:\left.f \mapsto \widehat{f}\right|_{\gamma}$, где $\gamma$ - так называемая модельная кривая в $\mathbb{R}^{n}$ вида $\left(t, t^{2}, \ldots, t^{n}\right)$.

Более того, в работах Хермандера [10] в случае $n=2$ и в многомерном случае в работе Бак и Лее [11] рассмотрены интегральные операторы следующего вида:

$$
T_{\lambda} f(x):=\int_{\mathbb{R}} e^{i \lambda \Phi(x, t)} a(x, t) f(t) d t,
$$

где $\Phi \in C^{\infty}\left(\mathbb{R}^{n} \times \mathbb{R}\right)$ - гладкая функция и $a \in C_{0}^{\infty}\left(\mathbb{R}^{n} \times \mathbb{R}\right)$ - гладкая функция, носитель которой сосредоточен в достаточно малой окрестности нуля и $f \in L^{p}(\mathbb{R})$.

В работе [11] получена оценка для нормы оператора $T_{\lambda}: L^{p}(\mathbb{R}) \mapsto L^{q}\left(\mathbb{R}^{n}\right)$ в виде $\left\|T_{\lambda}\right\|_{p, q}=O\left(\lambda^{-n / q}\right)$ при следующих достаточных условиях: 
a) $1 / p+n(n+1) /(2 q) \leqslant 1, q>n(n+1) / 2+1$;

b) отображение $\nabla \Phi(x, \cdot)$ определяет кривую с ненулевым кручением при любом фиксированном значении $x$ из носителя амплитуды.

Более того, доказана необходимость условия $1 / p+n(n+1) /(2 q) \leqslant 1$. Оказывается, необходимость условия $q>n(n+1) / 2+1$ связана с точным показателем суммируемости тригонометрических интегралов. Следует отметить, что из результатов работы [3] непосредственно вытекает точность оценок Друри [9], а также Бака и Лее [11] для модельных кривых, точнее, неограниченность "сопряженного" оператоpa $R^{*}: L^{p}(\gamma) \mapsto L^{q}\left(\mathbb{R}^{n}\right)$ при $q \leqslant n(n+1) / 2+1$. Следует отметить, что в этих работах утверждается неограниченность сопряженного оператора лишь для случая $n=2$.

В связи с этим естественно возникает задача: найти максимальное число $q_{0}$ такое, что при $q<q_{0}$ оператор $R^{*}$ неограничен для кривых с кручением и для более общих кривых конечного типа. Этими обстоятельствами объясняются мотивы поставленной задачи.

4. Обсуждение результатов. В настоящей работе мы получим решение задачи о точном показателе суммируемости для преобразования Фурье зарядов, сосредоточенных на кривых конечного типа. Из наших результатов следуют необходимые и достаточные условия ограниченности преобразования Фурье на гладких кривых конечного типа.

Оказывается, для кривых конечного типа показатель суммируемости преобразования Фурье зарядов естественно выражается в терминах инвариантов группы движений евклидова пространства [12].

Для полноты изложения приведем соответствующие определения [12]. Пусть $\gamma \subset$ $\mathbb{R}^{n}$ - некоторая дифференцируемая кривая. Ради определенности мы предположим, что $\varphi:[-1,1] \mapsto \mathbb{R}^{n}$ - некоторый параметр кривой.

Группа $C^{\infty}$-диффеоморфизмов отрезка $[-1,1]$ обозначается через $H$ и $G:=\mathbb{R}^{n} \otimes$ $O(n, \mathbb{R})$ - группа движений евклидова пространства.

Обозначим через $M$ множество дифференцируемых кривых пространства $\mathbb{R}^{n}$. В множестве кривых (путей) естественно действует группа $(G, H)$ по правилу

$$
\rho(g, h)(\varphi)(t):=g \varphi\left(h^{-1}(t)\right) .
$$

ОПРеДЕЛЕНИЕ. Отображение $\wp: M \mapsto \mathbb{R}$ называется относительным инвариантом (или ковариантом) веса $r$ группы $(G, H)$, если для любого элемента $(g, h) \in$ $(G, H)$ выполняется соотношение

$$
\wp(\rho(g, h)(\varphi))(t)=\left(\left(h^{-1}\right)^{\prime}(t)\right)^{r} \wp(\varphi)\left(h^{-1}\right)(t) .
$$

Введем следующие относительные инварианты:

$$
\Re(t):=\left(\varphi^{\prime}(t), \varphi^{\prime}(t)\right), \quad \Im(t)=\operatorname{det}\left[\varphi^{\prime}(t), \varphi^{\prime \prime}(t), \ldots, \varphi^{(n)}(t)\right] .
$$

Очевидно, что $\Re$ и $\Im$ являются относительными инвариантами веса 2 и $n(n+1) / 2$ соответственно.

Кривая $\gamma$ называется неособой, невырожденной (кривая с ненулевым кручением), если $\Im(t) \neq 0$ при любом $t \in[-1,1]$. Она называется кривой конечного типа в точке $\varphi\left(t_{0}\right)$, если $t_{0}$ не является корнем функции $\Im$ бесконечного порядка. В монографии [5] приведено определение конечности типа для произвольных поверхностей. Для кривых эти два определения эквивалентны. 
Очевидно, что если дифференцируемая кривая неособая, то она является гладкой, т.е. $\Re(t) \neq 0$ при любом $t \in[-1,1]$. Обратное, вообще говоря, неверно.

Легко показать, что вышеприведенные определения не зависят от выбора параметризации кривых.

Теорема 4.1. Пусть $\gamma \in \mathbb{R}^{n}$ - неособая кривая. Тогда при любом $q>n(n+1) / 2+1$ для преобразования Фуръе заряда $d \mu_{\alpha}(\xi)$ имеет место включение $\widehat{d \mu_{\alpha}(\xi)} \in L^{q}\left(\mathbb{R}^{n}\right)$. Более того, если $\psi(0) \neq 0$ и $\psi$ - гладкая функиия, сосредоточенная в достаточно малой окрестности нуля, то $\widehat{d \mu_{\alpha}(\xi)} \notin L^{q}\left(\mathbb{R}^{n}\right)$ nри $q \leqslant n(n+1) / 2+1$. В частности, для любого вещественного числа $\alpha$ имеем: $q(\gamma, \alpha)=n(n+1) / 2+1$.

Отметим, что точный показатель суммируемости $q(\gamma, \alpha)$ связан с весом относительного инварианта $\Im(t)$. Так как в этом случае кривая гладкая, то $\Re(t) \neq 0$ и, следовательно, точный показатель суммируемости не зависит от выбора числа $\alpha$.

Пусть $\Im(t)$ не имеет нулей бесконечного порядка. Тогда она имеет конечное число нулей на отрезке $[-1,1]$.

Введем следующее обозначение:

$$
q(\gamma, \alpha)=\max \left\{\frac{n(n+1)}{2}+1, \frac{2 m_{\Im}(t)+n(n+1)}{2\left(1+m_{\Re}(t) \alpha\right)}: m_{\Im}(t)=0\right\},
$$

где $m_{\Im}(t)\left(m_{\Re}(t)\right)$ - кратность корня $t$ функции $\Im(\Re)$ и $\alpha$ - произвольное вещественное число, удовлетворяющее условию: $1+m_{\Re}(t) \alpha>0$ для любого корня функции .

Теорема 4.2. Пусть $\gamma \in \mathbb{R}^{n}$ - кривая конечного типа. Тогда для любого $q>$ $q(\alpha, \gamma)$ мы имеем $d \mu_{\alpha} \in L^{q}\left(\mathbb{R}^{n}\right)$. Более того, если $t_{0} \in[-1,1]-$ некоторая точка, в которой выполняется равенство

$$
q(\gamma, \alpha)=\frac{2 m_{\Im}\left(t_{0}\right)+n(n+1)}{2\left(1+m_{\Re}\left(t_{0}\right) \alpha\right)}
$$

в случае $q(\gamma, \alpha)>n(n+1) / 2+1$ или условие

$$
\Im\left(t_{0}\right) \neq 0
$$

в случае $q(\gamma, \alpha) \leqslant n(n+1) / 2+1$, а также $\psi$ - гладкая функиия, удовлетворяющая условию $\psi\left(\varphi\left(t_{0}\right)\right) \neq 0$ и сосредоточенная в достаточно малой окрестности точки $\varphi\left(t_{0}\right)$, mo $\widehat{d \mu_{\alpha}} \notin L^{q(\alpha, \gamma)}\left(\mathbb{R}^{n}\right)$.

Как мы увидим из доказательств теорем 4.1 и 4.2, разница в формулах для точного показателя суммируемости связана с особенностями соответствующих фазовых функций. В случае особых кривых конечного типа вне особой точки фазовая функция является $\mathbb{R}_{+}$-версальной деформацией особенности, кратность которой строго меньше кратности начальной особой точки, а в случае неособой кривой фаза является $\mathbb{R}_{+}$-версальной деформацией особенности, но она не является миниверсальной (см. [13] для соответствующих определений). Это обстоятельство подтверждает гипотезу В.П. Паламодова. Он утверждал, что разница в формулах работы [3] для точного показателя суммируемости связана с условием "трансверсальности". 
Из теоремы 4.2 легко вытекает следующее утверждение.

СлеДСтвиЕ 4.3. Пусть $\gamma \in \mathbb{R}^{n}$ - кривая конечного типа. Тогда оператор

$$
R^{*} f(\xi):=\int_{\mathbb{R}} f(t) e^{2 \pi i(\xi, \varphi(t))}\left(\varphi^{\prime}(t), \varphi^{\prime}(t)\right)^{\alpha} \psi(\varphi(t)) d t
$$

не имеет тип $L^{p}(\mathbb{R}) \mapsto L^{q}\left(\mathbb{R}^{n}\right)$ для любого $q \leqslant q(\gamma, \alpha)$ при любом $p \geqslant 1$.

Пусть $T_{\lambda}$ - осцилляторный интегральный оператор вида (3.1), причем фазовая функция удовлетворяет следующему условию Карлесона-Сюлина [14]:

$$
\operatorname{det}\left(\partial_{t} \nabla \Phi(x, t), \partial_{t}^{2} \nabla \Phi(x, t), \ldots, \partial_{t}^{n} \nabla \Phi(x, t)\right) \neq 0 .
$$

Очевидно, что условие (4.1) означает, что при любом фиксированном значении $x$ отображение $\nabla \Phi(x, \cdot)$ определяет неособую кривую.

Из теоремы 4.1 и результатов Мокенхаупта [7], Бака и Ли [11] вытекает следующая

ТеоремА 4.4. Пусть $T_{\lambda}$ - семейство осиилляторных интегралъных операторов, определенное соотношением (3.1), и фазовая функиия $\Phi(x, t)$ удовлетворяет условию (4.1) на носителе амплитуды $a(x, t)$ и $a(0,0) \neq 0$. Тогда следующие условия эквивалентны:

1) $1 / p+n(n+1) /(2 q) \leqslant 1, q>n(n+1) / 2+1$;

2) существует постоянная $C$ такая, что при любых $f \in L^{p}(\mathbb{R})$ u $\lambda>1$ справедлива оченка

$$
\left\|T_{\lambda} f\right\|_{L^{q}\left(\mathbb{R}^{n}\right)} \leqslant C \lambda^{-n / q}\|f\|_{L^{p}(\mathbb{R})} .
$$

Теперь рассмотрим оценку интегральных операторов, ассоцированных с кривыми конечного типа. Пусть фазовая функция имеет вид

$$
\Phi(x, t)=x_{1} t^{k_{1}} b_{1}(t)+x_{2} t^{k_{2}} b_{2}(t)+\cdots+x_{n} t^{k_{n}} b_{n}(t),
$$

где $k_{1}<k_{2}<\cdots<k_{n}$ - натуральные числа и $b_{1}, b_{2}, \ldots, b_{n}$ - гладкие функции, удовлетворяющие условию: $b_{1}(0) b_{2}(0) \cdots b_{n}(0) \neq 0$.

Рассмотрим интегральный оператор

$$
T_{\lambda} f(x):=\int_{\mathbb{R}} e^{i \lambda \Phi(x, t)} a(t) f(t) d t .
$$

\section{Справедлива следующая}

Теорема 4.5. Пусть $T_{\lambda}$ - семейство осиилляторных интегралъных операторов, определенное соотношением (4.4), носитель амплитуды сосредоточен в достаточно малой окрестности нуля, $a(0) \neq 0$ и фазовая функция $\Phi(x, t)$ определена из равенства (4.3), причем $q_{0}:=k_{1}+k_{2}+\cdots+k_{n}>n(n+1) / 2$. Тогда следующие условия эквивалентны:

1) $1 / p+q_{0} / q \leqslant 1, q>q_{0}$;

2) существует постоянная $C$ такая, что при любых $f \in L^{p}(\mathbb{R})$ u $\lambda>1$ справедлива оценка (4.2). 
5. Суммируемость обобщенных максимальных функций Рендола. Пусть $f$ - гладкая функция, имеющая особенность типа $A_{n}$ (см. [13]) в начале координат $\mathbb{R}$ и $F(x, s, \sigma)$ - ее деформация. Рассмотрим осцилляторный интеграл

$$
J(\lambda, s, \sigma)=\int_{\mathbb{R}} a(x, s, \sigma) e^{i \lambda F(x, s, \sigma)} d x,
$$

где $\lambda$ - большой вещественный параметр и $a$ - гладкая функция с компактным носителем.

Отметим, что поведение осцилляторного интеграла (5.1) в зависимости от параметров $(\lambda, s, \sigma)$ может быть довольно сложным. Известны некоторые так называемые оценки с константами. Такие оценки естественно выражаются в терминах инвариантов классических групп (см. [15], [16]). Однако в некоторых задачах не требуются явные оценки "констант", а необходима их суммируемость. Оценки такого типа встречаются в классических работах Рендола [17] (также см. [5]).

Пусть $1 \leqslant \mu \leqslant n-$ фиксированное вещественное число. Введем "максимальную функцию" следующего вида:

$$
M_{\mu}(s, \sigma):=\sup _{\lambda \neq 0}|\lambda|^{1 /(\mu+1)}|J(\lambda, s, \sigma)| .
$$

Мы назовем ее обобщенной максимальной функиией Рендола. Рендол [17] ввел аналогичные максимальные функции для исследования поведения преобразования Фурье индикатора компактных, выпуклых областей с аналитической границей. Легко видеть, что для любого фиксированного числа $\mu$ максимальная функция $M_{\mu}$ является измеримой. Обобщенная лемма Ван дер Корпута показывает, что $M_{n} \in L^{\infty}[18]$. Случай $\mu=1$ был рассмотрен в работе [19] и показана, справедливость включения $M_{1} \in L_{\text {loc }}^{4-0}$, при условии версальности фазовой функции.

Введем обозначение

$$
q(n, \mu):=\frac{(\mu+1)\left(n^{2}+n-2\right)}{2(n-\mu)},
$$

а также для фиксированного $\mu, 1 \leqslant \mu \leqslant\left(n^{2}+2\right) /(2 n+1)$, определим

$$
q(\mu):=\min _{\mu \leqslant m \leqslant n} q(m, \mu) .
$$

Справедлива следующая

Теорема 5.1. Пусть $F(x, s, 0)$ является $\mathbb{R}_{+}$-версальной дебормацией особенности типа $A_{n}$. Существует окрестность нуля $U \times V \times W \subset \mathbb{R} \times \mathbb{R}^{m} \times \mathbb{R}^{l}$ такая, что при любой амплитудной функции $a \in C_{0}^{\infty}(U \times V \times W)$ для соответствующей максимальной функции $M_{\mu}$ имеют место следующие утверждения:

1) если $\mu \geqslant\left(n^{2}+2\right) /(2 n+1)$, то

$$
M_{\mu}(\cdot, \sigma) \in L^{q(n, \mu)-0}(V):=\bigcap_{q<q(n, \mu)} L^{q}(V),
$$

причем для любого $q<q(n, \mu)$ функиия $\left\|M_{\mu}(\cdot, \sigma)\right\|_{L^{q}(V)}$ ограничена на $W$;

2) если $\mu<\left(n^{2}+2\right) /(2 n+1)$, то

$$
M_{\mu}(\cdot, \sigma) \in L^{q(\mu)-0}(V),
$$

причем для любого $q<q(\mu)$ функция $\left\|M_{\mu}(\cdot, \sigma)\right\|_{L^{q}(V)}$ ограничена на $W$. 
Более того, результат точен в следующем смысле: если носитель амплитудь сосредоточен в достаточно малой окрестности нуля и $a(0,0) \neq 0$, то при $\mu \geqslant$ $\left(n^{2}+2\right) /(2 n+1)$ имеем

$$
M_{\mu}(\cdot, \sigma) \notin L^{q(n, \mu)}(V) \quad \text { для любого } \sigma \in W,
$$

а при $\mu<\left(n^{2}+2\right) /(2 n+1)$ имеем

$$
M_{\mu} \notin L^{q(\mu)}(V) \quad \text { для любого } \sigma \in W .
$$

ДОКАЗАТЕЛЬСТВО УТВЕРЖДЕНИЙ 1) и 2) ТЕОРЕМЫ 5.1. Заметим, что достаточно доказать утверждения теоремы 5.1 для любой фазовой функции, которая является версальной деформацией. В частности, без ограничения общности, мы можем предполагать, что $f(x)=x^{n+1}$ и $P(x, s)-\mathbb{R}_{+}$-версальная деформация, имеющая вид

$$
P(x, s)=x^{n+1}+s_{2} x^{n-1}+\cdots+s_{n} x,
$$

где $s=\left(s_{2}, s_{3}, \ldots, s_{n}\right) \in \mathbb{R}^{n-1} ;$ здесь индекс $j$ соответствует весу переменной $s_{j}$.

Введем класс амплитудных функций $A: a(x, s) \in A$, если

1) $a(x, s)$ - непрерывная функция от $(x, s) \in \mathbb{R} \times \mathbb{R}^{n-1}$;

2 ) для каждого фиксированного $s \in \mathbb{R}^{n-1}$ функция $a(\cdot, s)$ имеет ограниченную вариацию по $\mathbb{R}$;

3) полная вариация $V_{-\infty}^{\infty}[a(\cdot, s)]$ - локально-ограниченная функция. Рассмотрим осцилляторный интеграл

$$
J(\lambda, s)=\int_{\mathbb{R}} a(x, s) e^{i \lambda P(x, s)} d x,
$$

где $a(x, s) \in A$.

Легко видеть, что осцилляторный интеграл (5.3) условно сходится. Пусть $1 \leqslant$ $\mu \leqslant n$ и $m_{\mu}$ - максимальная функция, определенная по осцилляторному интегралу $J(\lambda, s)$.

Справедлива следующая

ЛЕмма 5.2. При любой амплитудной функиии $a \in A$ для соответствующей максимальной функиии $m_{\mu}$ имеют место следующие утверждения:

1) если $\mu \geqslant\left(n^{2}+2\right) /(2 n+1)$, mо $m_{\mu} \in L_{\text {loc }}^{q(n, \mu)-0}\left(\mathbb{R}^{n-1}\right)$;

2) если $\mu<\left(n^{2}+2\right) /(2 n+1)$, то $m_{\mu} \in L_{\text {loc }}^{q(\mu)-0}\left(\mathbb{R}^{n-1}\right)$.

Более того, если носитель амплитуды сосредоточен в достаточно малой окрестности нуля и $а(0,0) \neq 0$, то $m_{\mu}(\cdot, \sigma) \notin L_{\text {loc }}^{q(n, \mu)}\left(\mathbb{R}^{n-1}\right)$ nри $\mu \geqslant\left(n^{2}+2\right) /(2 n+1) u$ $m_{\mu} \notin L_{\mathrm{loc}}^{q(\mu)}\left(\mathbb{R}^{n-1}\right)$ npu $\mu<\left(n^{2}+2\right) /(2 n+1)$.

Лемма 5.2 доказывается методом индукции по $n$. Если $n=1$, то доказывать нечего. Если $n=2$, то мы имеем семейство максимальных функций $m_{\mu}$ с $1 \leqslant$ $\mu \leqslant 2$. В этом случае справедлива следующая явная оценка для осцилляторного интеграла [15]:

где

$$
|J(\lambda, s)| \leqslant \frac{c\|a(\cdot, s)\|_{1}}{|\lambda|^{1 / 4}+|\lambda|^{1 / 2}|s|^{1 / 4}},
$$

$$
\|a(\cdot, s)\|_{1}:=|a(-\infty, s)|+V_{\mathbb{R}}[a(\cdot, s)],
$$

$V_{\mathbb{R}}[a(\cdot, s)]$ - полная вариация функции $a(\cdot, s)$ по $\mathbb{R}$. 
Следовательно, для максимальной функции $m_{\mu}$ справедливо неравенство

$$
m_{\mu}(s) \leqslant \frac{c\|a(\cdot, s)\|_{1}}{|s|^{(2-\mu) /(2(\mu+1))}} .
$$

Заметим, что $q(n, \mu)=2(\mu+1) /(2-\mu)$.

Таким образом, в случае $n=2$ доказаны утверждения 1) и 2) леммы 5.2 о суммируемости максимальной функции.

Предположим, что $n>2$ - любое фиксированное натуральное число и утверждение 1$)$ леммы 5.2 справедливо для любого $l \leqslant n-1$. Иными словами, мы предположим, что $m_{\mu} \in L_{\text {loc }}^{q(\mu, l)-0}\left(\mathbb{R}^{n-1}\right)$ для каждого $\mu \geqslant\left(l^{2}+2\right) /(2 l+1)$, а также, что $m_{\mu} \in L_{\text {lос }}^{q(\mu)-0}\left(\mathbb{R}^{n-1}\right)$ для любого $\mu<\left(l^{2}+2\right) /(2 l+1)$, где $l \leqslant n-1$. Мы будем доказывать утверждение 1) леммы 5.2 для $n$, предполагая $\mu \geqslant\left(n^{2}+2\right) /(2 n+1)$.

Пусть

$$
\rho(s)=\left(\left|s_{2}\right|^{(n+1) ! / 2}+\left|s_{2}\right|^{(n+1) ! / 2}+\cdots+\left|s_{n}\right|^{(n+1) ! / n}\right)^{1 /(n+1) !} .
$$

Заметим, что $\rho(s)$ - квазиоднородная функция степени один с весами $(2,3, \ldots, n)$.

Обозначим через $\Sigma$ квазисферу $\Sigma:=\left\{s \in \mathbb{R}^{n-1}: \rho(s)=1\right\}$.

Если $K_{2}$ - фиксированное компактное множество, то для любого $s \in K_{2}$ множество критических точек функции $P(x, s)$ содержится в некотором компактном множестве $K_{1}$. В частности, мы можем взять в качестве $K_{2}$ любой компакт со свойством $\Sigma \subset K_{2}$.

Пусть $s=s^{0} \in \Sigma-$ фиксированная точка квазисферы $\Sigma$. Тогда фазовая функция $P\left(x, s^{0}\right)$ имеет конечное число критических точек $x_{1}, x_{2}, \ldots, x_{k} \in K_{1}$ кратности $l_{j} \leqslant n-1, j=1, \ldots, k$. Фиксируем $j$ и рассмотрим функцию $P(x, s)$ и осцилляторный интеграл $J_{j}(\lambda, s)$ с амплитудой, сосредоточенной в достаточно малой окрестности $U \times V$ точки $\left(x_{j}, s^{0}\right)$. Легко показать, что фазовая функция $P(x, s)$ [18], [19] является версальной деформацией особенности $A_{l_{j}}$. Вообще говоря, она не является миниверсальной деформацией. Если $\mu \geqslant l_{j}$, то классическая оценка типа Ван дер Корпута дает равномерную оценку относительно параметров $s \in V$ [3], (также см. [20]):

$$
\left|J_{j}(\lambda, s)\right| \leqslant \frac{\text { const }}{|\lambda|^{1 /(\mu+1)}}
$$

Далее, предположим, что $\mu<l_{j}$. Тогда, так как $\mu \geqslant\left(n^{2}+2\right) /(2 n+1)>\left(l_{j}^{2}+2\right) /$ $\left(2 l_{j}+1\right)$, согласно предположения индукции при $s \in V$ для интеграла $J_{j}(\lambda, s)$ имеем оценку

$$
\left|J_{j}(\lambda, s)\right| \leqslant \frac{\psi_{j}(s)}{|\lambda|^{1 /(\mu+1)}}, \quad \text { причем } \quad \psi_{j} \in L^{q\left(\mu, l_{j}\right)-0}(V) .
$$

Заметим, что для любых $\mu, n, l_{j}$ с $n \geqslant \mu \geqslant\left(n^{2}+2\right) /(2 n+1)>\left(l_{j}^{2}+2\right) /\left(2 l_{j}+1\right)$ справедливо неравенство $q\left(\mu, l_{j}\right)>q(\mu, n)$. Следовательно, имеет место включение $\psi_{j} \in L^{q(\mu, n)}(V)$. Мы получим аналогичные оценки для любого $j=1,2, \ldots, k$. Пусть $K_{0}$ - некоторая окрестность компакта $K_{1}$, содержащая множества критических точек функции $P(x, s)$ для любого $s \in V$. Если $J_{0}(t, s)$ - осцилляторный интеграл с амплитудой, сосредоточенной в $\mathbb{R} \backslash K_{0}$, то он имеет оценку $\left|J_{0}(\lambda, s)\right| \leqslant$ const $/|\lambda|^{1 /(\mu+1)}$. 
Следовательно, для интеграла $J(\lambda, s)$ получим

$$
|J(\lambda, s)| \leqslant \frac{\psi(s)}{|\lambda|^{1 /(\mu+1)}} \quad \text { с некоторой функцией } \quad \psi \in L^{q(\mu, n)}(V),
$$

где $\psi(s)=$ const $+\sum_{j=1}^{k} \psi_{j}(s)$.

Мы имеем оценку (5.4) для любой точки $s^{0} \in \Sigma$. Так как $\Sigma-$ компактное множество, существуют окрестность $V(\Sigma) \subset K_{2}$ и функция $\psi(s)$, удовлетворяющая условию $\psi \in L^{q(\mu, n)}(V(\Sigma))$; при этом для любой точки $s \in V(\Sigma)$ справедлива оценка

$$
|J(\lambda, s)| \leqslant \frac{\psi(s)}{|\lambda|^{1 /(\mu+1)}} .
$$

В частности, мы можем предполагать, что

$$
V(\Sigma)=\left\{s \in \mathbb{R}^{n-1}: 1-\varepsilon \leqslant \rho(s) \leqslant 1+\varepsilon\right\},
$$

где $\varepsilon$ - достаточно малое положительное число.

Рассмотрим диффеоморфное отображение $\Pi: \mathbb{R}^{n-1} \backslash\{0\} \mapsto \mathbb{R}_{+} \times \Sigma$, определенное равенством

$$
\Pi\left(s_{2}, s_{3}, \ldots, s_{n}\right):=\left(\rho(s), \frac{s_{2}}{\rho(s)^{2 /(n+1)}}, \frac{s_{3}}{\rho(s)^{3 /(n+1)}}, \ldots, \frac{s_{n}}{\rho(s)^{n /(n+1)}}\right) .
$$

Обозначим через $d s$ стандартную форму объема в $\mathbb{R}^{n-1} \backslash\{0\}$.

Лемма 5.3. Справедливо соотношение

$$
\Pi^{*}(d s)=\rho^{\left(n^{2}-n-3\right) /(n+1)} d \rho \wedge \omega,
$$

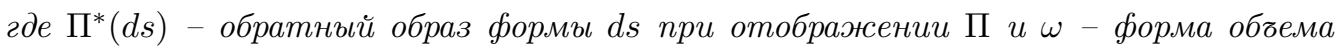
на $\Sigma$.

Лемма 5.3 доказывается стандартными методами анализа (см. [21], [19]).

Теперь возвратимся к доказательству леммы 5.2. Согласно теореме Фубини для почти всех точек $\rho \in(1-\varepsilon, 1+\varepsilon)$ существует следующий интеграл:

$$
\int_{\Sigma}\left(\psi\left(\Pi^{-1}(\rho, \sigma)\right)^{q(n, k)} \omega .\right.
$$

Ради определенности мы можем предполагать, что последный интеграл сходится при $\rho=1$. Легко показать, что для интеграла $J(\lambda, s)$ имеет место оценка [19]

$$
|J(\lambda, s)| \leqslant \frac{\psi\left(\Pi^{-1}(1, \sigma)\right)}{|\lambda|^{1 /(\mu+1)} \rho(s)^{(n-\mu) /((\mu+1)(n+1))}} .
$$

По нашему предположению

$$
\psi\left(\Pi^{-1}(1, \sigma)\right) \in L^{q(\mu, n)}(\Sigma) \quad \text { и } \quad \frac{1}{\rho(s)^{(n-\mu) /((\mu+1)(n+1))}} \in L_{\mathrm{loc}}^{q(\mu, n)-0}\left(\mathbb{R}^{n-1}\right) .
$$

Поэтому по известному следствию теоремы Фубини мы имеем включение

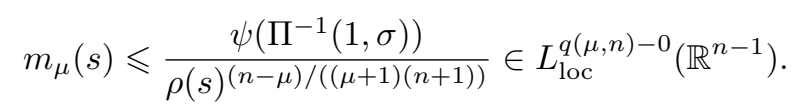


В случае $\mu<\left(n^{2}+2\right) /(2 n+1)$ суммируемость максимальной функции $M_{\mu}$ доказывается совершенно аналогично.

Таким образом, утверждения 1) и 2) леммы 5.2 доказаны. Из доказанного легко следует справедливость утверждений 1) и 2) теоремы 5.1.

6. Оценки снизу. В этом пункте мы получим некоторые оценки снизу для осцилляторных интегралов.

Рассмотрим осцилляторный интеграл (5.3) с фазовой функцией $P(x, s)$ и амплитудой $a \in C_{0}^{\infty}\left(\mathbb{R} \times \mathbb{R}^{n-1}\right)$. Предположим, что $\lambda$ - большой вещественный параметр и $s$ - малый параметры. Определим обобщенные функции Эйри [18]:

$$
Y_{j}(s):=\int_{\mathbb{R}} x^{j} e^{i P(x, s)} d x, \quad j=0,1, \ldots, n-1 .
$$

Интегралы (6.1) условно сходятся и являются вещественно аналитическими функциями от параметра $s \in \mathbb{R}^{n-1}$.

Известен, следующий классический результат [18].

Теорема 6.1. Существует окрестность нуля $V \times U \subset \mathbb{R} \times \mathbb{R}^{n-1}$ такая, что для любой амплитудной функции $a \in C_{0}^{\infty}(V \times U)$ для интеграла (5.3) справедливо следующее асимптотическое разложение:

$$
J(\lambda, s)=\sum_{j=0}^{n-1} a_{j}(s, \lambda) \lambda^{-(j+1) /(n+1)} Y_{j}\left(s_{2} \lambda^{2 /(n+1)}, \ldots, s_{n} \lambda^{n /(n+1)}\right)+O\left(|\lambda|^{-\infty}\right)
$$

при $|\lambda| \rightarrow+\infty$, где $O\left(|\lambda|^{-\infty}\right)$ означает справедливость соотношения

$$
J(\lambda, s)-\sum_{j=0}^{n-1} a_{j}(s, \lambda) \lambda^{-(j+1) /(n+1)} Y_{j}\left(s_{2} \lambda^{2 /(n+1)}, \ldots, s_{n} \lambda^{n /(n+1)}\right)=O\left(|\lambda|^{-N}\right)
$$

при $|\lambda| \rightarrow+\infty$ для любого фиксированного $N>0$, причем последнее соотношение выполняется равномерно относителъно $s$. Более того, $a_{j}(s, \lambda)$ имеет асимптотическое разложение вида

$$
a_{j}(s, \lambda) \approx \sum_{k=0}^{\infty} a_{j}^{k}(s) \lambda^{-k}, \quad j=0, \ldots, n-1
$$

Отметим, что если $a(0,0) \neq 0$, то $a_{0}^{0}(0,0) \neq 0$.

ПРЕДЛОЖЕНИЕ 6.2. Если $а(0,0) \neq 0$, то существуют положительные числа $\lambda_{0}$, $\varepsilon$, с такие, что для интеграла (5.3) имеет место следующая ощенка снизу:

$$
|J(\lambda, s)| \geqslant c /|\lambda|^{1 /(n+1)} \quad \text { npu } \quad|\lambda|>\lambda_{0},\left.\quad\left|s_{k}\right| \lambda\right|^{k /(n+1)} \mid<\varepsilon, \quad k=2, \ldots, n .
$$

ДокАЗАТЕЛЬСтво. Действительно, если $a(0,0) \neq 0$, то $a_{0}^{0}(0,0) \neq 0$, а также

$$
\operatorname{Re}\left(Y_{0}(0)\right)=2 \cos \left(\frac{\pi}{2(n+1)}\right) \Gamma\left(\frac{1}{n+1}\right) \neq 0
$$


где $\operatorname{Re}\left(Y_{0}(0)\right)$ - вещественная часть числа $Y_{0}(0)$ и $\Gamma$ - функция Эйлера. Так как $Y_{0}\left(s_{2}, \ldots, s_{n}\right)$ - гладкая функция, существует положительное число $\varepsilon>0$ такое, что для любых $\left(s_{2}, \ldots, s_{n}\right)$ с $\left|s_{k}\right|<\varepsilon, k=2, \ldots, n$, справедливо неравенство

$$
\left|Y_{0}\left(s_{2}, \ldots, s_{n}\right)\right| \geqslant c_{1}:=\cos \left(\frac{\pi}{2(n+1)}\right) \Gamma\left(\frac{1}{n+1}\right)>0 .
$$

Поэтому из разложения (6.2) получим следующую оценку снизу:

$$
|J(\lambda, s)| \geqslant c /|\lambda|^{1 /(n+1)} \quad \text { при } \quad|\lambda|>\lambda_{0},\left.\quad\left|s_{k}\right| \lambda\right|^{k /(n+1)} \mid<\varepsilon, \quad k=2, \ldots, n .
$$

где $c=a_{0}^{0}(0) c_{1} / 2$. Предложение 6.2 доказано.

Наконец, докажем утверждения леммы 5.2 и теоремы 5.1 о точности показателя суммируемости максимальных функций. Сначала рассмотрим осцилляторный интеграл

$$
I(\lambda, s)=\int_{\mathbb{R}} e^{i \lambda P(x, s)} h(x) d x,
$$

где $h \in C_{0}^{\infty}(\mathbb{R})$ - гладкая функция, удовлетворяющая условиям $0 \leqslant h(x) \leqslant 1$ и $h(x) \equiv 1$ в некоторой окрестности начала координат. Рассмотрим осцилляторный интеграл с амплитудой $h$ и фазовой функцией $P(x, s)=x^{n+1}+s_{2} x^{n-1}+\cdots+s_{n} x$.

Рассмотрим оценку снизу для соответствующей максимальной функции $m_{\mu}(s)$.

ЛЕмма 6.3. Для любого $1 \leqslant \mu \leqslant n$ в достаточно малой окрестности нуля справедлива следующая оценка снизу:

$$
m_{\mu}(s) \geqslant \frac{c}{\rho(s)^{(n-\mu) /(n+1)(\mu+1)}}
$$

где с - некоторое положительное число.

ДокАЗАТЕЛЬство. Сначала, рассмотрим случай $\mu=n$. Если $s$ лежит в достаточно малой окрестности нуля, то мы имеем соотношение

$J(\lambda, s)=\int_{\mathbb{R}} e^{i \lambda P(x, s)} d x+O\left(\lambda^{-\infty}\right)=\lambda^{-1 /(n+1)} Y_{0}\left(\lambda^{2 /(n+1)} s_{2}, \ldots, \lambda^{n /(n+1)} s_{n}\right)+O\left(\lambda^{-\infty}\right)$

при $\lambda \rightarrow+\infty$. Более того, последнее асимптотическое соотношение выполняется равномерно относительно параметров $s$.

Мы можем выбрать начало координат $V \subset \mathbb{R}^{n-1}$ и вещественное число $\lambda_{0}$ так, что для любого $\lambda>\lambda_{0}>1$ и $s \in V$ будет справедлива оценка

$$
\left|\int_{\mathbb{R}} e^{i \lambda P(x, s)} h(x) d x-\lambda^{-1 /(n+1)} Y_{0}\left(\lambda^{2 /(n+1)} s_{2}, \ldots, \lambda^{n /(n+1)} s_{n}\right)\right| \leqslant \frac{1}{\lambda} .
$$

С другой стороны, мы можем выбрать $V$ так, что при $s \in V$ будет выполняться неравенство $\left|Y_{0}(s)\right| \geqslant c>0$. Мы можем взять положительное число $\lambda_{0}$ и окрестность $V_{0} \subset V$ такие, что

$$
\lambda_{0}^{-n /(n+1)}<\frac{c}{3} \quad \text { и } \quad\left(\lambda_{0}^{2 /(n+1)} s_{2}, \ldots, \lambda_{0}^{n /(n+1)} s_{n}\right) \in V
$$


при $s \in V_{0}$. Тогда для любого $s \in V_{0}$ справедлива следующая оценка снизу:

$$
m_{n} \geqslant\left|Y_{0}\left(\lambda_{0}^{2 /(n+1)} s_{2}, \ldots, \lambda_{0}^{n /(n+1)} s_{n}\right)\right|-\frac{c}{3} \geqslant \frac{2 c}{3} .
$$

Пусть теперь $\mu, 1 \leqslant \mu<n$. Введем новый параметр $\eta$ и функцию

$$
\rho_{\eta}(s)=\left(\left|\eta s_{2}\right|^{(n+1) ! / 2}+\left|\eta s_{2}\right|^{(n+1) ! / 2}+\cdots+\left|\eta s_{n}\right|^{(n+1) ! / n}\right)^{1 /(n+1) !}
$$

и рассмотрим интеграл

$$
I(\lambda, s):=\int_{\mathbb{R}} e^{i \lambda P(x, s)} d x
$$

В последнем интеграле сделаем замену переменных $x=\rho_{\eta}(s)^{1 /(n+1)} y$ :

$$
I(\lambda, s):=\rho_{\eta}(s)^{1 /(n+1)} \int_{\mathbb{R}} e^{i \lambda \rho_{\eta}(s) P(x, \xi)} d x .
$$

Если $\eta$ - достаточно большое фиксированное положительное, то для каждой точки $s \in V_{0}$ мы можем выбрать параметр $\lambda$ так, что $\lambda \rho_{\eta}(s)=\lambda_{0}$. Для таких значений параметров справедливо следующее неравенство:

$$
m_{\mu}(s) \geqslant \frac{c \rho_{\eta}(s)^{1 /(n+1)}}{\rho_{\eta}(s)^{1 /(\mu+1)}}=c \rho_{\eta}(s)^{(\mu-n) /(\mu+1)(n+1)} .
$$

Заметим, что для любого фиксированного положительного числа $\eta$ имеем оценки

$$
c_{1} \rho_{\eta}(s) \leqslant \rho(s) \leqslant \rho_{\eta}(s) .
$$

Следовательно,

$$
m_{\mu}(s) \geqslant c \rho(s)^{(\mu-n) /(\mu+1)(n+1)} .
$$

Лемма 6.3 доказана.

Теперь возвратимся к доКАЗАТЕЛЬству лЕммы 5.2. Согласно лемме 6.3 получим $m_{\mu} \notin L_{\mathrm{loc}}^{q(n, \mu)}\left(\mathbb{R}^{n-1}\right)$ при $1 \leqslant \mu \leqslant n$. Более того, если $\mu<\left(n^{2}+2\right) /(2 n+1)$, то мы можем найти число $m \leqslant n$ такое, что $q(\mu)=q(m, \mu)$, а также мы можем найти $s^{0} \in \mathbb{R}^{n-1}$ и $x=x_{0} \in \mathbb{R}$ такие, что фазовая функция $P(x, s)$ является версальной деформацией особенности $A_{m}$ в точке $x_{0}$, а в остальных критических точках она имеет невырожденные особенности. Поэтому, $m_{\mu} \notin L_{\mathrm{loc}}^{q(\mu)}\left(\mathbb{R}^{n-1}\right)$. Лемма 5.2 доказана.

Из леммы 5.2 легко следует справедливость теоремы 5.1.

7. Случай неособых кривых. В этом пункте приведем доказательство теоремы 4.1. Прежде доказываются некоторые вспомогательные утверждения.

ПРеДЛОЖЕНИЕ 7.1. Пусть $\gamma \subset \mathbb{R}^{n}$ - кривая конечного типа в начале координат. Тогда существуют единственным способом определенные натуральные числа $1 \leqslant k_{1}<k_{2}<\cdots<k_{n}$ такие, что кривая $\gamma$ вращением системы координат может быть записана в виде

$$
\varphi(t)=\left(t^{k_{1}} b_{1}(t), t^{k_{2}} b_{2}(t), \ldots, t^{k_{n}} b_{n}(t)\right),
$$

где $b_{1}, b_{2}, \ldots, b_{n}$ - гладкие функции такие, что $b_{1}(0) b_{2}(0) \cdots b_{n}(0) \neq 0$. 
Набор чисел $\left(k_{1}, k_{2}, \ldots, k_{n}\right)$ называется типом кривой $\gamma$ в начале координат.

ДоКАЗАТЕЛЬСТво предложения 7.1 очевидно.

ДоКАЗАТЕЛЬСТво тЕОРЕмЫ 4.1. Если $\gamma$ - неособая кривая, то без ограничения общности мы можем считать, что

$$
\varphi(t)=\left(t b_{1}(t), \ldots, t^{n-1} b_{n-1}, \frac{t^{n}}{n}\right)
$$

где $b_{1}, \ldots, b_{n-1}$ - гладкие функции, удовлетворяющие условию $b_{1}(0) \cdots b_{n-1}(0) \neq 0$; при этом носитель функции $\psi(x)$ содержится в достаточно малой окрестности начала координат.

Отметим, что в этом случае $s_{d}(t) \neq 0$. Поэтому, полагая $\alpha=0$, достаточно исследовать поведение следующего интеграла:

$$
\widehat{d \mu}(\xi)=\int_{\mathbb{R}} e^{2 \pi i(\xi, \varphi(t))} \psi(\varphi(t)) d t .
$$

Основной вклад в этот интеграл дает достаточно малая окрестность начала координат в случае, когда вектор $\xi$ находится в достаточно малой конической окрестности точки $(0, \ldots, 1)$. В этой окрестности координаты вектора $\xi$ удовлетворяют условиям: $\delta\left|\xi_{n}\right|>\left|\xi_{j}\right|, j=1, \ldots, n-1$, где $\delta$ - фиксированное достаточно малое положительное число. Заметим, что фазовая функция $(\xi, \varphi(t))$ имеет более вырожденную особенность именно в направлении вектора $(0, \ldots, 0,1)$. Вне окрестности этой точки фазовая функция является деформацией особенности $A_{k}, k \leqslant n-2$.

Запишем функцию $(\xi, \varphi(t))$ в виде

$$
(\xi, \varphi(t))=\xi_{n}\left(\frac{t^{n}}{n}+\frac{\xi_{n-1}}{\xi_{n}} t^{n-1} b_{n-1}(t)+\cdots+\frac{\xi_{1}}{\xi_{n}} t b_{1}(t)\right):=\xi_{n} \Phi(t, \sigma),
$$

где

$$
\Phi(t, \sigma)=\frac{t^{n}}{n}+\sigma_{n-1} t^{n-1} b_{n-1}(t)+\cdots+\sigma_{1} t b_{1}(t), \quad \sigma_{n-1}=\frac{\xi_{n-1}}{\xi_{n}}, \quad \ldots, \quad \sigma_{1}=\frac{\xi_{1}}{\xi_{n}} .
$$

Справедливо следующее утверждение.

ПрЕДЛОЖЕНИЕ 7.2. Функиия $\Phi(t, \sigma)-\mathbb{R}_{+}$-версалъная деформация функции $t^{n} / n$.

ДоКАЗАТЕЛЬСТво ПРЕДЛОЖЕНИЯ 7.2 легко следует из теоремы Мозера о версальности [13].

Теперь докажем суммируемость интеграла $\widehat{d \mu}(\xi)$. Пусть $\nu=(n-1) / 2+1 / n+\varepsilon$, где $\varepsilon-$ достаточно малое положительное число. Заметим, что $\left((n-1)^{2}+2\right) /(2 n-1)<\nu$.

Пусть $q$ - любое вещественное число, удовлетворяющее условию

$$
\frac{(n+1) n}{2}+1+n \varepsilon<q<\frac{(n+1) n+2+2 n \varepsilon)\left(n^{2}-n-2\right)}{2\left(n^{2}-n-2-2 n \varepsilon\right)} .
$$

Покажем справедливость соотношения $\widehat{d \mu} \in L^{q}\left(\mathbb{R}^{n}\right)$. 
Предположим, что $\delta\left|\xi_{n}\right|>\left|\xi_{k}\right|, k=1,2, \ldots, n-1$, где $\delta$ - достаточно малое положительное число. Как отметили выше, это наиболее вырожденное направление. Согласно теореме 5.1 выполнено неравенство

$$
|\widehat{d \mu}(\xi)| \leqslant M_{\nu}\left(\frac{\xi_{n-1}}{\xi_{n}}, \ldots, \frac{\xi_{1}}{\xi_{n}}\right)\left|\xi_{n}\right|^{-1 /(\nu+1)},
$$

причем $M_{\nu} \in L^{q}(V)$, где $V$ - некоторая достаточно малая окрестность начало координат $\mathbb{R}^{n-1}$. Также, при $n(n+1) / 2+1+(n+1) \varepsilon<q$ имеем $q /(\nu+1)-n>0$. Поэтому $\widehat{d \mu} \in L^{q}(\Omega(\delta))$, где $\Omega(\delta) \subset \mathbb{R}^{n}$ - множество, определенное неравенствами $\delta\left|\xi_{n}\right|>\left|\xi_{k}\right|, k=1,2, \ldots, n-1$.

Случай $\delta\left|\xi_{n}\right| \leqslant\left|\xi_{k}\right|, k=1,2, \ldots, n-1$, рассматривается аналогично. Таким образом, имеет место включение $\widehat{d \mu} \in L^{q}\left(\mathbb{R}^{n}\right)$.

Теперь, докажем точность результата.

ПреДЛОЖЕНИЕ 7.3. Предположим, что $\psi \in C_{0}^{\infty}(-\delta, \delta) u \psi(0) \neq 0$. Тогда $\widehat{d \mu} \notin$ $L^{q}\left(\mathbb{R}^{n}\right)$ для любого $q \leqslant(n+1) n / 2+1$.

Приведем известное утверждение [22].

ЛЕмма 7.4. Существует гладкое отображение

$$
\Psi:(-\delta, \delta) \times V(0) \mapsto\left(\varepsilon_{1}, \varepsilon_{2}\right) \times \mathbb{R}^{n-1},
$$

где $\varepsilon_{1}<0<\varepsilon_{2}$, имеющее вид

$$
\left(Y\left(t, \sigma_{1}, \ldots, \sigma_{n-1}\right), s_{1}(\sigma), s_{2}(\sigma), \ldots, s_{n-1}(\sigma)\right)
$$

u maкое, чmo

$$
\Phi\left(t\left(Y, \sigma_{1}, \ldots, \sigma_{n-1}\right), \sigma_{1}, \ldots, \sigma_{n-1}\right)=Y^{n}+s_{2} Y^{n-1}+\cdots+s_{n-1} Y+s_{1}(\sigma),
$$

где $s_{1}, s_{2}, \ldots, s_{n-1}$ - гладкие функции с условиями $s_{l}(0)=0, l=2, \ldots, n-1$, причем $\operatorname{rang}(D S(0))=n-2$, где $D S(0)-$ дифференциал отображения $S:=\left(s_{2}(\sigma), \ldots\right.$, $\left.s_{n-1}(\sigma)\right)$ в нуле.

Лемма 7.5. Пусть $F_{1}, F_{2}, \ldots, F_{n-1}$ - гладкие функиии, удовлетворяющие условию $\operatorname{rang}(D F)(0)=n-1 u$

$$
\Omega:=\left\{x \in U \subset \mathbb{R}^{n}:\left|F_{k}(x)\right|<\varepsilon_{k}, k=1, \ldots, n-1\right\},
$$

где $U$ - достаточно малая окрестность начала координат в $\mathbb{R}^{n}$. Тогда для лебеговой меры множества $\Omega$ имеем

$$
|\Omega|=C(\varepsilon) \prod_{k=1}^{n-1} \varepsilon_{k}
$$

где $C(\varepsilon)$ - некоторая функиия, удовлетворяющая следующему условию: существуют положительные числа $c_{1}, c_{2}$ такие, что $c_{1} \leqslant C(\varepsilon) \leqslant c_{2}$ для достаточно ма$л b x \varepsilon$.

Лемму 7.5 легко доказать, записывая меру множества $\Omega$ в виде интеграла. 
ДОКАЗАТЕЛЬСТвО ПРЕДЛОЖЕНИЯ 7.3. Рассмотрим множество

$$
\Omega_{\delta}:=\left\{\xi: \delta\left|\xi_{n}\right| \geqslant\left|\xi_{j}\right|, j=1, \ldots, n-1\right\}
$$

где $\delta$ - достаточно малое положительное число.

Пусть $s_{j}(\sigma), j=2, \ldots, n-1,-$ гладкие функции из леммы 7.4. Если $c$ - достаточно малое положительное число и

$$
\Omega_{0}=\left\{\xi \in \mathbb{R}^{n}: \xi_{n}>M,\left|s_{2}(\sigma)\right|<c\left|\xi_{n}\right|^{-2 / n}, \ldots,\left|s_{n-1}(\sigma)\right|<c\left|\xi_{n}\right|^{-(n-1) / n}\right\} \subset \Omega_{\delta},
$$

то согласно предложению 6.2 для любого $\xi \in \Omega_{0}$ имеем следующую оценку снизу: $|\widehat{\mu}(\xi)| \geqslant c /\left|\xi_{n}\right|^{1 / n}$, где $c-$ фиксированное положительное число.

Применяя лемму 7.5 при $q \leqslant n(n+1) / 2+1$, получим

$$
\int_{\Omega_{\delta}}|\widehat{\mu}(\xi)|^{q} d \xi \geqslant \int_{\Omega_{0}}\left|\widehat{\mu}\left(\xi_{n}, \sigma\right)\right|^{q} \xi_{n}^{n-1} d \xi_{n} d \sigma \geqslant c \int_{M}^{\infty} \xi_{n}^{(n-1) / 2-(q-1) / n} d \xi_{n}=\infty .
$$

Предложение 7.3 доказано.

Предложение 7.3 показывает, что если $\psi(0) \neq 0$, то выполнено $\widehat{d \mu} \notin L^{q}\left(\mathbb{R}^{n}\right)$ при $q \leqslant n(n+1) / 2+1$. Что и завершает доказательство теоремы 4.1.

ЗАмЕчаниЕ 7.6. Пусть $\gamma(\sigma)$ - семейство гладких кривых, где $\sigma \in V \subset \mathbb{R}^{m}$ - некоторая окрестность нуля. Оно определяется параметризацией $\varphi(t, \sigma):[-1,1] \mapsto \mathbb{R}^{n}$. Рассмотрим соответствующий заряд $d \mu(\sigma):=\psi(\varphi(t, \sigma)) s_{d}(t, \sigma)^{1 / 2} d t$. Доказательство теоремы 4.1 показывает, что если $\gamma(0)$ - неособая кривая и $V \subset \mathbb{R}^{m}$ - достаточно малая окрестность нуля, то для любого $q>n(n+1) / 2+1$ справедливо включение $\widehat{d \mu(\sigma)} \in L^{q}\left(\mathbb{R}^{n}\right)$, причем для любого фиксированного $q>n(n+1) / 2+1$ функция $\|\widehat{d \mu(\sigma)}\|_{L^{q}\left(\mathbb{R}^{n}\right)}$ ограничена на $V$.

8. Кривые конечного типа. Этот пункт посвящен доказательству теоремы 4.2.

Пусть $\gamma-$ кривая типа $\left(k_{1}, k_{2}, \ldots, k_{n}\right)$ в нуле и $d \mu_{\alpha}$ - заряд, имеющий вид $d \mu_{\alpha}(t):=$ $\psi(\varphi(t))|t|^{\alpha} d t$, где $\alpha>-1-$ некоторое фиксированное вещественное число и $\psi \in$ $C_{0}^{\infty}(U)$; здесь $U$ - достаточно малая окрестность начала координат. Ради упрощения обозначений здесь мы слегка изменили вид заряда. Применяя предложения 7.1 и используя соответствующее разбиение единицы, достаточно доказать теорему 4.2 для этого частного случая.

Таким образом, мы можем предполагать, что

$$
\gamma(t)=\left(t^{k_{1}} b_{1}(t), t^{k_{2}} b_{2}(t), \ldots, t^{k_{n}} b_{n}(t)\right)
$$

и $b_{1}(0) b_{2}(0) \cdots b_{n}(0) \neq 0$. Докажем справедливость включения $\widehat{d \mu_{\alpha}} \in L^{q}\left(\mathbb{R}^{n}\right)$ при $q>q(\alpha)$, где

$$
q(\alpha)=\max \left\{\frac{n(n+1)}{2}+1, \frac{2 m_{\Im}(0)+n(n+1)}{2(1+\alpha)}\right\} .
$$

Предположим, что $\beta \in C_{0}^{\infty}((-2,-1 / 2) \cup(1 / 2,2))$ - гладкая функция со свойствами [6]

$$
0 \leqslant \beta \leqslant 1, \quad \sum_{j=0}^{\infty} \beta\left(2^{j} t\right)=1, \quad t \in \operatorname{supp}\{\psi\} \backslash\{0\} .
$$


Используя это разбиение единицы, можем записать $\widehat{d \mu_{\alpha}}(\xi)$ в виде суммы следующих интегралов:

$$
\widehat{d \mu_{j}}(\xi):=\int_{\mathbb{R}} e^{2 \pi i F(\xi, t)} \psi(\varphi(t))|t|^{\alpha} \beta\left(2^{j} t\right) d t .
$$

Если носитель функции $\psi$ находится в достаточно малой окрестности нуля, то существует достаточно большое число $N$ такое, что при $j \leqslant N$ имеем $\frac{d_{\mu}}{d \mu_{j}}(\xi) \equiv 0$. Поэтому мы можем считать, что $j \gg 1$.

Используя замену переменных $2^{j} t \mapsto t$, получим

$$
\widehat{d \mu_{j}}(\xi):=2^{-j(1+\alpha)} \int_{\mathbb{R}} e^{2 \pi i F_{j}(\eta, t)} \psi\left(2^{-j} t\right)|t|^{\alpha} \beta(t) d t,
$$

где

$$
\eta_{1}:=2^{-j k_{1}} \xi_{1}, \ldots, \eta_{n}:=2^{-j k_{n}} \xi_{n}, \quad F_{j}(\eta, t):=\eta_{n} t^{k_{n}} b_{n}\left(2^{-j} t\right)+\cdots+\eta_{1} t^{k_{1}} b_{1}\left(2^{-j} t\right) .
$$

Для определенности предположим, что

$$
\left|\eta_{n}\right|=\max \left\{\left|\eta_{1}\right|,\left|\eta_{2}\right|, \ldots,\left|\eta_{n}\right|\right\} .
$$

Запишем функцию $F_{j}(\eta, t)$ в виде

$F_{j}(\eta, t)=\eta_{n}\left(t^{k_{n}} b_{n}\left(2^{-j} t\right)+\zeta_{n-1} t^{k_{n-1}} b_{n-1}\left(2^{-j} t\right)+\cdots+\zeta_{1} t^{k_{1}} b_{1}\left(2^{-j} t\right)\right):=\eta_{n} P(t, \zeta)$.

Обозначим через $\Sigma$ множество $\left\{\zeta: \max _{1 \leqslant j \leqslant n-1}\left|\zeta_{j}\right| \leqslant 1\right\}$. Фиксируем $\zeta:=\zeta^{0} \in \Sigma$.

Лемма 8.1. Предположим, что фазовая функиия

$$
P_{0}\left(t, \zeta^{0}\right):=t^{k_{n}} b_{n}(0)+\zeta_{n-1} t^{k_{n-1}} b_{n-1}(0)+\cdots+\zeta_{1} t^{k_{1}} b_{1}(0)
$$

имеет критическую точку $t=t^{0} \in(-2,-1 / 2) \cup(1 / 2,2)$. Тогда для кратности $m\left(t^{0}\right)$ этой критической точки имеем $m:=m\left(t^{0}\right) \leqslant n-1$. Более того, $P_{0}(t, \zeta)$ является версальной деформачией особенности $A_{m}$ в точке $\left(t^{0}, \zeta^{0}\right)$.

ДокАЗАТЕЛЬСтво. Предположим, что $l \leqslant n-1$ - число ненулевых координат $\zeta^{0} \in \mathbb{R}^{n-1}$. Тогда легко видеть, что система уравнений

$$
P^{\prime}\left(t^{0}, \zeta^{0}\right)=\cdots=P^{(l+1)}\left(t^{0}, \zeta^{0}\right)=0
$$

не имеет ненулевых решений относительно $t^{0}$. Следовательно, хотя бы одна из производных полинома $P_{0}\left(t, \zeta^{0}\right)$ порядка $j \leqslant l+1$ отлична от нуля в точке $t^{0} \in$ $(-2,-1 / 2) \cup(1 / 2,2)$.

Пусть фазовая функция $P_{0}\left(t, \zeta^{0}\right)$ в точке $t^{0} \in(-2,-1 / 2) \cup(1 / 2,2)$ имеет особенность типа $A_{l}$. Тогда функции (векторы) $\pi\left(t^{k_{1}}\right), \pi\left(t^{k_{2}}\right), \ldots, \pi\left(t^{k_{l-1}}\right)$ линейно независимы в пространстве $\mathscr{M} / I_{\left(t-t^{0}\right)^{l}}$, где $\mathscr{M}$ - идеал, состоящий из гладких функций, обращающихся в нуль в точке $t^{0}, \mathscr{M} / I_{\left(t-t^{0}\right)^{l}}[13]$ - локальная алгебра особенности в точке $t=t^{0} \neq 0$ и $\pi$ - естественный гомоморфизм $\pi: C^{\infty}(\mathbb{R}) \mapsto \mathscr{M} / I_{\left(t-t^{0}\right)^{l}}$. Теперь, используя теорему версальности Мозера [13], получим, что $P_{0}(t, \zeta)$ является $\mathbb{R}_{+}$-версальной деформацией особенности $A_{l}$. Лемма 8.1 доказана. 
ДокАЗАТЕЛЬСТво теОРемы 4.2. Согласно теореме 4.1 с учетом замечания 7.6 получим: если $q>n(n+1) / 2+1$, то следующий интеграл сходится и он равномерно ограничен относительно $j$ :

$$
\int_{\mathbb{R}^{n}}\left|\int_{\mathbb{R}} e^{2 \pi i F_{j}(t, \eta)} \psi\left(2^{-j} t\right) \beta(t) d t\right|^{q} d \eta \leqslant C .
$$

Отсюда для функции $\widehat{d \mu_{j}}(\xi)$ имеем

$$
\left\|\widehat{d \mu_{j}}(\xi)\right\|_{L^{q}\left(\mathbb{R}^{n}\right)} \leqslant C 2^{j\left(q_{0} / q-(1+\alpha)\right)}, \quad \text { где } \quad q_{0}:=k_{1}+\cdots+k_{n} .
$$

Поэтому ряд $\sum_{j=0}^{\infty} \widehat{d \mu_{j}}(\xi)$ сходится в пространстве $L^{q}\left(\mathbb{R}^{n}\right)$ при $q>q(\alpha)$ и его сумма $\widehat{d \mu_{\alpha}}$ принадлежит $L^{q}\left(\mathbb{R}^{n}\right)$ для таких $q$.

Точность результатов. Необходимость условия $q>n(n+1) / 2+1$ легко следует из оценки снизу, использованной в доказательстве теоремы 4.1. Потому будем предполагать, что $q_{0} /(1+\alpha)>n(n+1) / 2+1$.

Предположим, что $0 \leqslant h(t) \leqslant 1-$ гладкая функция с компактным носителем и $h(t) \equiv 1$ в некоторой окрестности начала координат. Если $h(t)-$ произвольная гладкая функция и $h(0) \neq 0$, то задача об оценке снизу легко сводится к этому случаю. Рассмотрим осцилляторный интеграл

$$
J(\lambda, \sigma):=\int h(t)|t|^{\alpha} e^{i \lambda\left(t^{k_{n}}+\sigma_{1} t^{k_{1}} b_{1}(t)+\cdots+\sigma_{n-1} t^{k_{n-1}} b_{n-1}(t)\right)} d t .
$$

ПРЕДЛОЖЕНИЕ 8.2. Если $q_{0} /(1+\alpha) \geqslant n(n+1) / 2+1$, то существуют положительные числа $\lambda_{0}, \varepsilon$, с такие, что при

$$
\lambda>\lambda_{0}, \quad\left|\sigma_{l}\right| \lambda^{\left(k_{n}-k_{l}\right) / k_{n}}<\varepsilon, \quad l=1, \ldots, n-1,
$$

для интеграла $J(\lambda, \sigma)$ выполняется следующая оценка снизу:

$$
|J(\lambda, \sigma)| \geqslant \frac{c}{\lambda^{(\alpha+1) / k_{n}}} .
$$

В интеграле $J(\lambda, \sigma)$ сделаем замену переменных $t=\lambda^{-1 / k_{n}} \tau$ и, обозначая новую переменную снова через $t$, получим

$J(\lambda, \sigma):=\lambda^{-(\alpha+1) / k_{n}} \int_{\mathbb{R}} h\left(\lambda^{-1 / k_{n}} t\right)|t|^{\alpha} e^{i\left(t^{k_{n}}+\widetilde{\sigma}_{1} t^{k_{1}} b_{1}\left(\lambda^{-1 / k_{n}} t\right)+\cdots+\widetilde{\sigma}_{n-1} t^{k_{n-1}} b_{n-1}\left(\lambda^{-1 / k_{n}} t\right)\right)} d t$, где

$$
\widetilde{\sigma}_{1}=\sigma_{1} \lambda^{\left(k_{n}-k_{1}\right) / k_{n}}, \quad \ldots, \quad \widetilde{\sigma}_{n-1}=\sigma_{n-1} \lambda^{\left(k_{n}-k_{n-1}\right) / k_{n}} .
$$

Теперь рассмотрим следующие вспомогательные интегралы:

$$
J_{1}(\lambda, \tilde{\sigma}):=\lambda^{(\alpha+1) / k_{n}} J(\lambda, \sigma), \quad J_{2}(\widetilde{\sigma})=\int_{\mathbb{R}}|t|^{\alpha} e^{i\left(t^{k_{n}}+\widetilde{\sigma}_{1} t^{k_{1}} b_{1}(0)+\cdots+\widetilde{\sigma}_{n-1} t^{k_{n-1}} b_{n-1}(0)\right)} d t .
$$

Фазовые функции осцилляторных интегралов $J_{1}(\lambda, \widetilde{\sigma})$ и $J_{2}(\widetilde{\sigma})$ обозначаются через $F_{1}(t, \lambda, \widetilde{\sigma})$ и $F_{2}(t, \widetilde{\sigma})$ соответственно. Здесь переменные $\lambda$ и $\widetilde{\sigma}$ рассматриваются как независимые.

Амплитуда интеграла $J_{1}(\lambda, \tilde{\sigma})$ при каждом фиксированном значении параметра $\lambda$ имеет компактный носитель. Интеграл $J_{2}(\widetilde{\sigma})$ условно сходится и является гладкой функцией от параметров $\widetilde{\sigma}$. 
ЛЕмма 8.3. Интеграл $J_{1}(\lambda, \widetilde{\sigma})$ локально равномерно сходится $\kappa$ интегралу $J_{2}(\widetilde{\sigma})$ nрu $\lambda \rightarrow+\infty$.

ДокАЗАТЕЛЬСтво. Пусть $C$ - любое фиксированное положительное число. Для любого положительного числа $\varepsilon$ существуют положительное число $r$ и гладкая функция $0 \leqslant \chi(t) \leqslant 1$ такие, что $\chi(t)=0$ при $|t| \leqslant r$ и $\chi(t)=1$ при $|t| \geqslant r+1$, причем для любого вектора $\widetilde{\sigma}$ из множества $\{|\widetilde{\sigma}| \leqslant C\}$ выполняется неравенство: $\left|J_{2}(\widetilde{\sigma})\right|<\varepsilon$.

Кроме того, мы можем считать, что $h\left(\lambda^{-1 / k_{n}} t\right)=1$ при $|t| \leqslant r+1$ и $\lambda>\lambda_{0}$.

Заметим, что если $q_{0} /(1+\alpha) \geqslant n(n+1) / 2+1$, то мы имеем неравенство $k_{n} /(1+\alpha)>$ $3 / 2$. С помощью формулы интегрирования по частям можно показать справедливость следующей оценки для любых $\lambda>\lambda_{0}$ и $|\widetilde{\sigma}| \leqslant C$ :

$$
\left.\left|\int_{\mathbb{R}}(1-\psi(t)) h\left(\lambda^{-1 / k_{n}} t\right)\right| t\right|^{\alpha} e^{i F_{1}(t, \lambda, \tilde{\sigma})} d t \mid<\varepsilon .
$$

Теперь рассмотрим оценку интеграла

$$
\int_{\mathbb{R}} \psi(t)\left(e^{i F_{1}(t, \lambda, \widetilde{\sigma})}-e^{i F_{2}(t, \tilde{\sigma})}\right) d t .
$$

Легко видеть, что в последнем интеграле подынтегральная функция равномерно по $|t| \leqslant r+1$ и $|\widetilde{\sigma}| \leqslant C$ сходится к нулю при $\lambda \rightarrow+\infty$. Отсюда легко следует доказательство леммы 8.3 .

ДОКАЗАТЕЛЬСТВо ПРЕДЛОЖЕНИЯ 8.2. Как отметили, $J_{2}(\widetilde{\sigma})$ - непрерывная функция и $J_{2}(0) \neq 0$. Следовательно, существует положительное число $\varepsilon$ такое, что при любом $|\widetilde{\sigma}|<\varepsilon$ выполняется неравенство $\left|J_{2}(\widetilde{\sigma})\right| \geqslant\left|J_{2}(0)\right| / 2$. С учетом леммы 8.3 получим существование положительных чисел $\lambda_{0}, \varepsilon, c$ таких, что при

$$
\lambda>\lambda_{0}, \quad\left|\sigma_{l}\right| \lambda^{\left(k_{n}-k_{l}\right) / k_{n}}<\varepsilon, \quad l=1, \ldots, n-1,
$$

для интеграла $J(\lambda, \sigma)$ выполняется следующая оценка снизу:

$$
|J(\lambda, \sigma)| \geqslant \frac{1}{\lambda^{(\alpha+1) / k_{n}}}\left(\left|J_{2}(\widetilde{\sigma})\right|-\left|J_{1}(\lambda, \widetilde{\sigma})-J_{2}(\widetilde{\sigma})\right|\right) \geqslant \frac{\left|J_{2}(0)\right|}{3 \lambda^{(\alpha+1) / k_{n}}} .
$$

Что и требовалось доказать.

Окончание доКАЗАТЕЛЬСТВА тЕОРЕмы 4.2. Аналогично доказательству теоремы 4.1 рассмотрим множество

$$
\Omega_{0}=\left\{\xi \in \mathbb{R}^{n}: \xi_{n}>M,\left|\xi_{l}\right|<\varepsilon\left|\xi_{n}\right|^{k_{l} / k_{n}}, l=1, \ldots, n-1\right\},
$$

где $M(\varepsilon)$ - достаточно большое (малое) фиксированное положительное число. Согласно предложению 8.2 для любого $\xi \in \Omega_{0}$ имеем следующую оценку снизу:

$$
\left|\widehat{d \mu_{\alpha}}\right| \geqslant \frac{c}{\left|\xi_{n}\right|^{(\alpha+1) / k_{n}}},
$$

где $c$ - фиксированное положительное число.

Далее, используя замену переменных $\xi_{j}=\sigma_{j} \xi_{n}, j=1, \ldots, n$, при $q \leqslant q_{0} /(1+\alpha)$ получим

$$
\int_{\mathbb{R}^{n}}\left|\widehat{d \mu_{\alpha}}\right|^{q} d \xi \geqslant \int_{\Omega_{0}}\left|\widehat{d \mu_{\alpha}}\right|^{q} \xi_{n}^{n-1} d \xi_{n} d \sigma \geqslant c \int_{M}^{\infty} \xi_{n}^{n-1+\left(q_{0}-n k_{n}\right) / k_{n}-(\alpha+1) q / k_{n}} d \xi_{n}=\infty .
$$

Последнее соотношение завершает доказательство теоремы 4.2. 


\section{9. Об ограничении преобразования Фурье на кривых и оценки соответ- ствующих осцилляторных интегральных операторов.}

ДокАЗАТЕЛЬСТво тЕОРЕмЫ 4.4. Мокенхаупт показал, что если для осцилляторного интегрального оператора (3.1) справедлива оценка (4.2), то для любой точки $x^{0} \in \pi(\operatorname{supp}(a))\left(\right.$ где $\pi: \mathbb{R}^{n} \times \mathbb{R} \mapsto \mathbb{R}^{n}$ - естественный проектор) следующий осцилляторный интеграл:

$$
\int_{\mathbb{R}} a\left(x^{0}, t\right) e^{i\left(\xi, \nabla \Phi\left(x^{0}, t\right)\right.} d t
$$

принадлежит $L^{q}\left(\mathbb{R}^{n}\right)$. Отсюда следует, что если $a(0,0) \neq 0$ и фазовая функция удовлетворяет условиям (4.1), то согласно теореме 4.1 для справедливости оценки (4.2) необходимо условие $q>n(n+1) / 2+1$. Необходимость условия $1 / p+n(n+1) /(2 q) \leqslant 1$ и достаточность условия $q>n(n+1) / 2+1$ доказаны в работе [11]. Теорема 4.4 доказана.

ДокаЗАтЕЛЬСтво теоремы 4.5. Рассмотрим функцию $\beta \in C_{0}^{\infty}((-2,-1 / 2) \cup$ $(1 / 2,2))$ и соответствующую разбиению единицы, как в доказательстве теоремы 4.2. Используя это разбиение единицы, получим

$$
T_{\lambda} f=\sum_{j=0}^{\infty} T_{\lambda}^{j} f, \quad \text { где } \quad T_{\lambda}^{j} f:=\int_{\mathbb{R}} e^{i \lambda \Phi(x, t)} a(t) \beta\left(2^{j} t\right) f(t) d t .
$$

В интеграле $T_{\lambda}^{j} f$ сделаем замену переменных $2^{j} t \mapsto t$ и получим

$$
T_{\lambda}^{j} f:=2^{-j} \int_{\mathbb{R}} e^{i \lambda \Phi_{j}(y, t)} a\left(2^{-j} t\right) \beta(t) f_{j}(t) d t,
$$

где $f_{j}(t):=f\left(2^{-j} t\right) \chi(t), \chi(t)-$ характеристическая функция носителя функции $\beta$ и

$$
\Phi_{j}(y, t):=y_{1} t^{k_{1}} b_{1}\left(2^{-j} t\right)+y_{2} t^{k_{2}} b_{2}\left(2^{-j} t\right)+\cdots+y_{n} t^{k_{n}} b_{n}\left(2^{-j} t\right),
$$

где $y_{l}:=2^{-j k_{l}} x_{l}, l=1, \ldots, n$.

Если носитель амплитуды $a$ находится в достаточно малой окрестности нуля, то мы можем считать, что $j \gg 1$ и, следовательно, фазовая функция $\Phi_{j}(y, t)$ может быть рассмотрена как малая деформация следующей функции:

$$
\Phi_{\infty}(y, t):=y_{1} t^{k_{1}} b_{1}(0)+y_{2} t^{k_{2}} b_{2}(0)+\cdots+y_{n} t^{k_{n}} b_{n}(0) .
$$

Отметим, что $\nabla \Phi_{\infty}(0, t)$ является неособой кривой на носителе функции $\beta$. Заметим, в случае, когда фазовая функция удовлетворяет условию (4.1), верхняя оценка (4.2) для нормы оператора выполняется равномерно относительно малых возмущений фазы [11]. Поэтому согласно теореме 4.4 получим

$$
\begin{aligned}
\left\|T_{\lambda}^{j} f\right\|_{L^{q}\left(\mathbb{R}_{x}^{n}\right)}^{q} & \leqslant \lambda^{-n} 2^{-j q+j q_{0}} \int_{\mathbb{R}^{n}}\left|\int_{\mathbb{R}} e^{i \lambda \Phi_{j}(y, t)} a\left(2^{-j} t\right) \beta(t) f_{j}(t) d t\right|^{q} d y \\
& \leqslant c \lambda^{-n} 2^{-j q+j q_{0}+j q / p}\|f\|_{L^{p}(\mathbb{R})}^{q},
\end{aligned}
$$

где $(p, q)$ - любая пара вещественных чисел, удовлетворяющих условиям $1 / p+$ $n(n+1) / 2 q \leqslant 1, q>n(n+1) / 2+1$. 
Отметим, что если $1 / p+q_{0} / q<1$, то искомая оценка легко следует суммированием ряда по $j$.

Но нетривиальный случай $1 / p+q_{0} / q=1$ требует некоторую интерполяционную технику.

Справедливо следующее

ПРЕДЛОЖЕНИЕ 9.1. Для любых чисел $r, s \in[1, \infty]$, удовлетворяющих условиям $1 / r+q_{0} / s=1$ us $>q_{0}$, существует постоянная с такая, что для любой функции $f \in L^{r, 1}(\mathbb{R})$ справедлива оченка

$$
\left\|T_{\lambda} f\right\|_{L^{s, \infty}\left(\mathbb{R}^{n}\right)} \leqslant C \lambda^{-n / s}\|f\|_{L^{r, 1}(\mathbb{R})},
$$

где $L^{s, \infty}\left(\mathbb{R}^{n}\right)$ и $L^{r, 1}(\mathbb{R})$ - соответствующие пространства Лоренца.

ДокАзАтельство. Случай $r=1, s=\infty$ тривиален. Пусть $(r, s)$ - любая пара, удовлетворяющая условиям $1 / r+q_{0} / s=1, r>1$ и $s>q_{0}$.

Заметим, что если $s>q_{0}$, то по условию $s>n(n+1) / 2$.

Следовательно, если $\varepsilon$ - достаточно малое положительное число, то для чисел

$$
r_{ \pm}:=\frac{r}{1 \pm \varepsilon}, \quad s_{ \pm}:=\frac{r}{1 \pm \varepsilon}
$$

выполняются условия

$$
\frac{1}{r_{ \pm}}+\frac{n(n+1)}{2 s_{ \pm}} \leqslant 1, \quad s_{ \pm}>\frac{n(n+1)}{2}+1
$$

Следовательно, согласно теореме 4.4 для любого $j$ (см. [11]) справедливы оценки

$$
\left\|T_{\lambda}^{j} f\right\|_{L^{s_{ \pm}, \infty}\left(\mathbb{R}^{n}\right)} \leqslant C \lambda^{-n / s_{ \pm}} 2^{ \pm \varepsilon j}\|f\|_{L^{r_{ \pm}, 1}(\mathbb{R})} .
$$

Отсюда с применением леммы 2.3 работы [11] получим требуемую оценку (9.1). Предложение 9.1 доказано.

Из предложения 9.1 с применением вещественной интерполяции [23] легко следует искомая оценка (4.2).

Теперь докажем необходимость условий $1 / p+q_{0} / q \leqslant 1$ и $q>q_{0}$ при $a(0) \neq 0$. Необходимость условия $q>q_{0}$ следует из теоремы 4.2 (с $\left.\alpha=0\right)$ и из результатов работы [7].

Необходимость условия $1 / p+q_{0} / q \leqslant 1$ легко следует из рассуждений работы [11]. Действительно, возьмем достаточно малое положительное число $\delta$ и рассмотрим функцию $f_{\delta}(t):=\chi_{[0, \delta]}(t)$, где $\chi_{[0, \delta]}$ - характеристическая функция отрезка $[0, \delta]$. Определим множество

$$
\Omega(\delta):=\left\{x \in \mathbb{R}^{n}:\left|x_{l}\right| \leqslant c \delta^{-k_{l}}\right\},
$$

где $c$ - достаточно малое фиксированное положительное число. Тогда получим

$$
c_{1}\|f\|_{L^{p}(\mathbb{R})}^{q}=c_{1} \delta^{q / p} \geqslant\left\|T_{1} f_{\delta}\right\|_{L^{q}\left(\mathbb{R}^{n}\right)}^{q} \geqslant \int_{\Omega(\delta)}\left|T_{1} f_{\delta}\right|^{q} d x \geqslant c_{2} \delta^{-q_{0}+q},
$$

где $c_{1}, c_{2}$ - некоторые фиксированные положительные числа. Отсюда для любого положительного числа $\delta$ получим неравенство $c_{3} \leqslant \delta^{1 / p+q_{0} / q-1}$ с некоторым фиксированным положительным числом $c_{3}$, что и доказывает необходимость условия $1 / p+q_{0} / q \leqslant 1$. Теорема 4.5 полностью доказана. 
Справедливо следующее утверждение об ограничении преобразования Фурье на кривых конечного типа.

СЛЕДСТВИЕ 9.2. Пусть $\gamma \subset \mathbb{R}^{n}$ - гладкая кривая конечного типа в начале координат, $m_{\Im}(0)>0 u d \mu=\psi(x) d l-$ борелева мера с $\psi(0) \neq 0$. Тогда оператор ограничения

$$
R f(t):=\widehat{f} \circ \varphi(t)
$$

где $f \in S\left(\mathbb{R}^{n}\right), S\left(\mathbb{R}^{n}\right)$ - пространства Швариа, имеет тип $L^{q}\left(\mathbb{R}^{n}\right) \mapsto L^{p}(\gamma)$ тогда и только тогда, когда

$$
q<\frac{q_{0}}{q_{0}-1}, \quad p \leqslant \frac{q}{q_{0}(q-1)},
$$

где

$$
q_{0}=m_{\Im}(0)+\frac{n(n+1)}{2} .
$$

Заметим, что в этом случае $m_{\Im}(0) \neq 0$ и результат отличается сдвигом на единицу от случая, когда $\gamma \subset \mathbb{R}^{n}-$ неособая кривая.

В заключение автор выражает свою глубокую благодарность профессору Дж. Г. Баку, обратившему внимание автора на эти задачи, а также Ш. А. Алимову за полезные обсуждения полученных результатов.

\section{СПИСОК ЦИТИРОВАННОЙ ЛИТЕРАТУРЫ}

[1] И. М. Виноградов, Метод тригонометрических сумм в теории чисел, 2-е изд., испр. и доп., Наука, М., 1980.

[2] L.-K. Hua, "On the number of solutions of Tarry's problem", Acta Sci. Sinica, 1:1 (1952), $1-76$.

[3] Г. И. Архипов, А. А. Карацуба, В.Н. Чубариков, "Тригонометрические интегралы", Изв. АН СССР. Сер. матем., 43:5 (1979), 971-1003.

[4] I. A. Ikromov, "On the convergence exponent of trigonometric integrals", Аналитическая теория чисел и приложения, Сборник статей. К 60-летию со дня рождения профессора Анатолия Алексеевича Карацубы, Тр. МИАН, 218, Наука, М., 1997, 179-189.

[5] E. M. Stein, Harmonic Analysis. Real-Variable Methods, Orthogonality, and Oscillatory Integrals, Princeton Math. Ser., 43, Princeton Univ. Press, Princeton, NJ, 1993.

[6] C. D. Sogge, Fourier Integrals in Classical Analysis, Cambridge Tracts in Math., 105, Cambridge Univ. Press, Cambridge, 1993.

[7] G. Mokenhaupt, Bounds in Lebesgue Spaces of Oscillatory Integral Operators, Habilitationsschrift, Universität Siegen, 1996.

[8] M. Christ, "On the restriction of the Fourier transform to curves: endpoint results and the degenerate case", Trans. Amer. Math. Soc., 287:1 (1985), 223-238.

[9] S. W. Drury, "Degenerate curves and harmonic analysis", Math. Proc. Cambridge Philos. Soc., 108:1 (1990), 89-96.

[10] L. Hörmander, "Oscillatory integrals and multipliers on FL", Ark. Mat., 11 (1973), 1-11.

[11] J.-G. Bak, S. Lee, "Estimates for an oscillatory integral operator related to restriction to space curves", Proc. Amer. Math. Soc., 132:5 (2004), 1393-1401.

[12] Дж. Хаджиев, Приложения теории инвариантов $\kappa$ дифберенииальной геометрии кривых, ФАН, Ташкент, 1988.

[13] В. И. Арнольд, А.Н.Варченко, С.М.Гусейн-заде, Особенности дифферениируемых отображений, т. 1, Классификация критических точек, каустик и вольновых фронтов, Наука, М., 1982. 
[14] L. Carleson, P. Sjölin, "Oscillatory integrals and a multiplier problem for the disc", Studia Math., 44 (1972), 287-299.

[15] И. А. Икромов, "Инвариантные оценки двумерных тригонометрических интегралов", Матем. сб., 180:8 (1989), 1017-1032.

[16] Д. А. Попов, "Восстановление характеристических функций в двумерной радоновской томографии", УМН, 53:1 (1998), 115-198.

[17] B. Randol, "On the asymptotic behavior of the Fourier transform of the indicator function of a convex set", Trans. Amer. Math. Soc., 139 (1969), 279-285.

[18] J. Duistermaat, "Oscillatory integrals, Lagrange immersions and unfolding of singularities", Comm. Pure Appl. Math., 27:2 (1974), 207-281.

[19] И. А. Икромов, "Об оценке преобразования Фурье индикатора невыпуклых областей”, Функи. анализ и его прил., 29:3 (1995), 16-24.

[20] Y. Domar, "On the Banach algebra $A(G)$ for smooth sets $\Gamma \subset \mathbb{R}^{n}$ ", Comment. Math. Helv., 52:3 (1977), 357-371.

[21] В.Н. Карпушкин, "Равномерные оценки осциллирующих интегралов с параболической или гиперболической фазой", Тр. сем. им. И. Г. Петровского, 9, Изд-во Моск. ун-та, М., 1983, 3-39.

[22] В. Гийемин, С. Стернберг, Геометрические асимптотики, Мир, М., 1981.

[23] И. Стейн, Г. Вейс, Введение в гармонический анализ на евклидовых пространствах, Мир, М., 1974.

И. А. Икромов

Поступило

Самаркандский государственный университет

29.12.2008

им. Алишера Навои

E-mail: ikromov1@rambler.ru 